\title{
Breakdown of universality in transitions to spatiotemporal chaos
}

Bohr, Tomas; Hecke, Martin van; Mikkelsen, René; Ipsen, Mads

Published in:

Physical Review Letters

Link to article, DOI:

10.1103/PhysRevLett.86.5482

Publication date:

2001

Document Version

Publisher's PDF, also known as Version of record

Link back to DTU Orbit

Citation (APA):

Bohr, T., Hecke, M. V., Mikkelsen, R., \& Ipsen, M. (2001). Breakdown of universality in transitions to spatiotemporal chaos. Physical Review Letters, 86(24), 5482-5485.

https://doi.org/10.1103/PhysRevLett.86.5482

\section{General rights}

Copyright and moral rights for the publications made accessible in the public portal are retained by the authors and/or other copyright owners and it is a condition of accessing publications that users recognise and abide by the legal requirements associated with these rights.

- Users may download and print one copy of any publication from the public portal for the purpose of private study or research.

- You may not further distribute the material or use it for any profit-making activity or commercial gain

- You may freely distribute the URL identifying the publication in the public portal

If you believe that this document breaches copyright please contact us providing details, and we will remove access to the work immediately and investigate your claim 


\title{
Breakdown of Universality in Transitions to Spatiotemporal Chaos
}

\author{
Tomas Bohr, ${ }^{1}$ Martin van Hecke, ${ }^{2}$ René Mikkelsen, ${ }^{2}$ and Mads Ipsen ${ }^{3}$ \\ ${ }^{1}$ Department of Physics, The Technical University of Denmark, DK-2800 Kgs. Lyngby, Denmark \\ ${ }^{2}$ Center for Chaos and Turbulence Studies, The Niels Bohr Institute, Blegdamsvej 17, DK-2100, Copenhagen Ø, Denmark \\ ${ }^{3}$ Fritz-Haber-Institut der Max-Planck-Gesellschaft, Faradayweg 4-6, D-14195 Berlin, Germany
}

(Received 17 August 2000)

\begin{abstract}
We show that the transition from laminar to active behavior in extended chaotic systems can vary from a continuous transition in the universality class of directed percolation with infinitely many absorbing states to what appears as a first-order transition. The latter occurs when finite lifetime nonchaotic structures, called "solitons," dominate the dynamics. We illustrate this scenario in an extension of the deterministic Chaté-Manneville coupled map lattice model and in a soliton including variant of the stochastic Domany-Kinzel cellular automaton.
\end{abstract}

DOI: $10.1103 /$ PhysRevLett.86.5482

The nature of transitions in extended deterministic dynamical systems is not very well understood. Since few analytical methods are available for such systems, it is tempting to map them to stochastic models [1]. In a study of the deterministic "damped Kuramoto-Sivashinsky equation" [2], Chaté and Manneville [3] introduced the notion of a universal transition to turbulence via "spatiotemporal intermittency" [4,5]. Pomeau gave general arguments [6] for this transition to be in the universality class of directed percolation (DP) $[7,8]$. These arguments rested on earlier work by Grassberger [9] and Janssen [10], who conjectured that any stochastic process with a unique absorbing state should be in the class of DP. To check whether deterministic models could be in the DP class, Chaté and Manneville introduced a very simple coupled map lattice (CML), with the local map either performing "laminar" or chaotic motion.

Surprisingly, the critical exponents of this CML were not those of DP [11]; in fact, they appeared to vary continuously along the critical line. Grassberger and Schreiber [12] pointed out that the presence of long lived "solitons" (local excitations that propagate with unit velocity through the lattice; see Figs. 1b and 1c and [12]) may lead to long crossover times and conjectured that the true asymptotic behavior of the Chate-Manneville model would be in the DP universality class.

In this Letter, we show that even solitons with a finite lifetime can completely change the nature of the transition. We base this claim on studies of extensions of (i) the Chaté-Manneville model [3] and (ii) the Domany-Kinzel stochastic cellular automaton [13], where both extensions facilitate the tuning of solitonic properties. In the regime of short soliton lifetimes the bulk exponents of our CML are consistent with DP; the spreading exponents indicate that the CML falls in the universality class of DP with infinitely many absorbing states. For both our CML and our stochastic model the transition between active and inactive states loses its continuous nature and appears to become first order for large soliton lifetimes. We argue that this discontinuity emerges because pairs of solitons can gen-
PACS numbers: 05.45.+b, 05.70.Fh, 47.27.Cn

erate new activity upon collisions (see Figs. 1c and 4a) while individual solitons do not lead to new turbulent activity. We study this phenomenon in detail in our stochastic cellular automaton and show that the nonuniversality seen in earlier work is likely due to the proximity of this (quasi-)first-order transition: our stochastic model shows transient behavior which can be fitted quite convincingly to nonuniversal power laws. Here we focus on the broad picture; a detailed study will be presented elsewhere [14].

Coupled map lattice.-The model introduced by Chaté and Manneville [11] is a 1D coupled map lattice

$$
u_{i}(n+1)=f\left(u_{i}(n)\right)+\frac{\varepsilon}{2} \Delta_{f} u_{i}(n),
$$

where $\Delta_{f} u_{i}(n)=f\left(u_{i-1}(n)\right)-2 f\left(u_{i}(n)\right)+f\left(u_{i+1}(n)\right)$. When $u \leq 1, f$ is a standard tent map of the form $f(u)=$ $r\left(\frac{1}{2}-\left|u-\frac{1}{2}\right|\right)$ and $u$ displays chaotic behavior, while when $u \geq 1, f$ is simply the identity and $u$ displays laminar behavior. States in which all sites are laminar remain so, but chaotic sites can "infect" their neighbors due to the spatial coupling. The effectiveness of this spreading of the chaos depends on $r$ and $\varepsilon$. Taking the density of chaotic sites or "activity" $m$ as an order parameter, transitions from a laminar state (in which $m$ decays to zero) to a "turbulent" state (where $m$ reaches a finite value in an infinite system) can be defined. To relate such CML's to physical systems

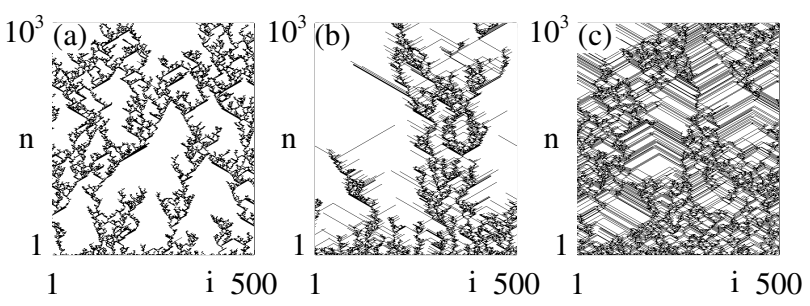

FIG. 1. Spacetime plots of our coupled map lattice (2)-(3) where inactive (chaotic) sites are white (black) for $r=3$ near criticality, illustrating the increasing role of solitons as a function of our parameter $b$ : (a) $b=0.2, \varepsilon=0.373$; (b) $b=0$ (ChatéManneville), $\varepsilon=0.357$; (c) $b=-0.1, \varepsilon=0.352$. 
of weakly coupled chaotic elements, we must interpret the map $f$ as a return map on a Poincaré section; each local map should therefore be at least two dimensional and invertible [15]. We thus introduce the CML

$$
\begin{aligned}
& u_{i}(n+1)=f\left(u_{i}(n)\right)+\frac{\varepsilon}{2} \Delta_{f} u_{i}(n)+v_{i}(n), \\
& v_{i}(n+1)=b\left(u_{i}(n+1)-u_{i}(n)\right),
\end{aligned}
$$

where $f$ is the same as before and the new parameter $b$ is the Jacobian of the local map at each site; this map is invertible for any nonzero $b$ and becomes increasingly two dimensional with $|b|$. This design is analogous to the construction of the Hénon map [16] from the logistic map, except that on the right hand side $b\left(u_{i}(n+1)-u_{i}(n)\right)$ appears instead of $b u_{i}(n)$. This ensures that the absorbing state fixed points $u_{i}(n)=u^{*}$ of the old CML (1) are mapped to the laminar fixed point $\left(u_{i}(n), v_{i}(n)\right)=\left(u^{*}, 0\right)$. The model is updated synchronously and typical dynamical states are shown in Fig. 1; in what follows we fix the parameter $r=3$.

To get a feeling for the location and nature of the transition we show in Fig. 2a the activity after 1000 time steps as a function of $b$ and $\varepsilon$; the active and inactive phases can be clearly distinguished. In Fig. $2 \mathrm{~b}$ we show that when $b$ is varied, there is a qualitative difference in the activity as a function of $\varepsilon$. The behavior for $b=0$ is consistent with a continuous transition, whereas for $b=-0.1$ a marked steepening occurs consistent with the emergence of a discontinuity; further support for this comes from a study of the correlation function which shows a finite correlation length at the transition [14]. In Fig. 2c we show the sharpening of this discontinuity as a function of the total integration time.

$C M L$ near continuous transition.-We focus now on the soliton-poor regime where the transition appears to be continuous. We have computed critical exponents using finite size scaling $[1,17]$. The critical line in the parameter plane $(\varepsilon, r)$ is located by measuring the "absorption time" $\tau(r, \varepsilon, L)$, i.e., the average time it takes the system, starting from a random initial state, to reach the absorbing state. At the critical point $\varepsilon=\varepsilon_{c}(r)$, this time diverges
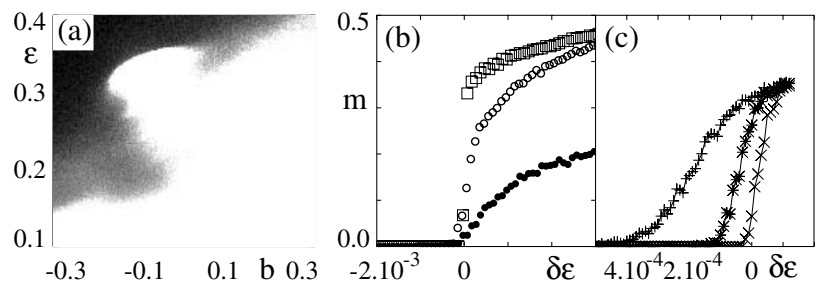

FIG. 2. Activity in the model (2)-(3) for a system size of 2048 and ensemble of 128 runs. (a) Activity at $t=1000$ (white $=$ inactive). (b) Activity as a function of $\delta \varepsilon$ (distance to critical point) at time $2 \times 10^{5}$ for $b=-0.1$ (squares), $b=0$ (open circles), and $b=0.2$ (closed circles). (c) Illustration of the sharpening of the $b=-0.1$ transition for increasing times $5 \times 10^{3}(+), 5 \times 10^{4}(*)$, and $5 \times 10^{5}(\times)$; note the strongly magnified scale. like $\tau\left(\varepsilon_{c}, L\right) \sim L^{z}$ where the usual dynamical exponent $z=\nu_{\|} / \nu_{\perp}$ has been introduced. The order parameter $m(\varepsilon, L, t)$ is the fraction of chaotic sites in the lattice, again averaged over many different initial states. The scaling of the order parameter, $m \sim\left(\varepsilon-\varepsilon_{c}\right)^{\beta}$ for $\varepsilon \rightarrow \varepsilon_{c}^{+}$, defines the critical exponent $\beta$. Precisely at the critical point the order parameter decays as $m\left(\varepsilon_{c}, t, L\right) \sim L^{-\beta / \nu_{\perp}} g\left(t / L^{z}\right)$. For $t \ll L^{z}$ this behavior should be independent of $L$ and $m\left(\varepsilon_{c}, t, L\right) \sim t^{-\theta}$ with $\theta=\beta / \nu_{\|}$. Table I shows the exponents $z$ and $\theta$ as a function of $b$ for $r=3$. These values were obtained for system sizes up to $L=2048$ (128 realizations) [14]. There are apparently regimes for $|b|>$ 0.1 where the exponents are very close to their DP values.

Spreading properties of our CML.- Instead of following the decay of an initially uniformly filled system, one can also determine critical exponents from the spreading of an initial seed of turbulence in an otherwise laminar configuration [19]. These so-called dynamical exponents are defined in terms of the number of chaotic sites $N(t)$, the survival probability $P(t)$, the mean-squared deviation $R^{2}(t)$ from the origin of the turbulent activity, and the density $n(t)$ of chaotic sites within the spreading patch of turbulence. Thus we assume

$$
N(t) \sim t^{\eta} \quad P(t) \sim t^{-\delta} \quad R^{2}(t) \sim t^{z_{s}} \quad n(t) \sim t^{-\theta_{s}} .
$$

For DP one finds $\delta=\theta=\theta_{s}$ and $z_{s}=2 / z$. In the ChatéManneville model at $r=3$ it is basically impossible to determine these exponents, since the spreading is completely dominated by the solitons (Fig. 3b). In our generalized CML it turns out to be possible to determine the spreading exponents (Fig. 3c) in the weak soliton regime. In the CML the absorbing state is nonunique since any state in which all $u$ values are above unity and all $v$ values are not too large is absorbing. We indeed found the dynamical exponents to depend on the configuration of the laminar sites; the spreading exponents are nonuniversal. This is consistent with recent studies of systems with infinite numbers of absorbing states [18,20-24] where it has been

TABLE I. The critical exponents $z$ and $\theta=\beta / \nu_{\|}$for our CML (2)-(3). Note that $\varepsilon_{c}(b)$ is a multiple-valued function and the values for $b>-0.1$ correspond to the "upper branch" of Fig. 2a. The values for DP (last row) are taken from [18].

\begin{tabular}{llll}
\hline \hline \multicolumn{1}{c}{$b$} & \multicolumn{1}{c}{$\epsilon_{c}$} & \multicolumn{1}{c}{$z$} & \multicolumn{1}{c}{$\theta$} \\
\hline-0.25 & $0.16312(3)$ & $1.58(1)$ & \\
-0.2 & $0.16495(2)$ & $1.58(2)$ & $0.168(2)$ \\
-0.15 & $0.16205(1)$ & $1.58(1)$ & $0.17(1)$ \\
-0.125 & $0.16368(2)$ & $1.57(1)$ & $0.20(1)$ \\
-0.1 & $0.35203(1)$ & $1.52(3)$ & $0.02(2)$ \\
0 & $0.35984(3)$ & $1.42(2)$ & $0.18(1)$ \\
0.1 & $0.3393(1)$ & $1.48(2)$ & $0.155(1)$ \\
0.125 & $0.34745(5)$ & $1.53(2)$ & $0.15(1)$ \\
0.15 & $0.35680(5)$ & $1.57(1)$ & $0.159(3)$ \\
0.175 & $0.36545(1)$ & $1.58(1)$ & $0.16(1)$ \\
0.2 & $0.37323(1)$ & $1.58(1)$ & $0.16(1)$ \\
DP & & $1.58074(4)$ & $0.15947(3)$ \\
\hline \hline
\end{tabular}



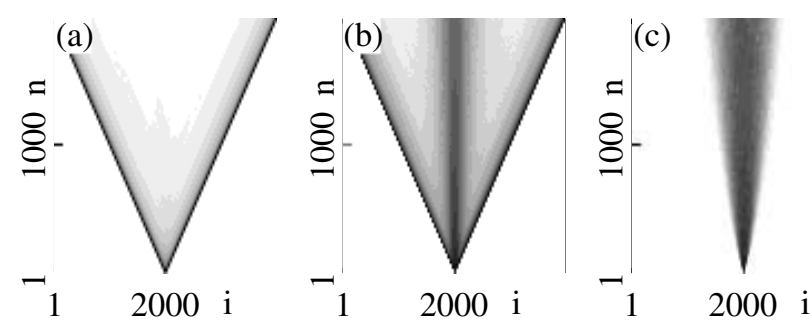

FIG. 3. Spreading of activity in our CML averaged over $10^{4}$ realizations for $b=-0.1$ (a), $b=-0$ (b), and $b=0.2$ (c).

revealed that these differ from classical DP precisely in the nonuniversality of the spreading exponents. It has also been conjectured, and verified numerically $[21,22]$, that the dynamical exponents satisfy the hyperscaling relation $\eta+\delta+\theta_{s}=d z_{s} / 2$, where $d$ is the spatial dimension. The spreading exponents depend on the mean value of the field $\langle u\rangle$ in the laminar state outside of the seed. For the example shown in Fig. 3c, the value of $\langle u\rangle$ which would result from a long run with homogeneously random initial conditions is 1.235 . Even a $2 \%$ variation in $\langle u\rangle$ creates a $100 \%$ variation of $\eta$, whereas $\Delta \equiv z_{s} / 2-$ $\left(\eta+\delta+\theta_{s}\right)$ remains less than 0.1 (for times up to 2000). Similarly, within this range $\delta$ varies between 0 and 0.5 . For other parameter values $\eta$ may range from 0.8 to 0.1 ; for more details, see [14].

First-order behavior and stochastic model.-The cause of the discontinuity in the activity in the soliton-rich regime is illustrated in spacetime plots as in Fig. 1c: while individual turbulent patches clearly have a finite lifetime, new activity is created by collisions of the solitons that surround the active patches. To illustrate this scenario, we extend the Domany-Kinzel cellular automaton [13] in the following way: (i) Our model contains two species representing the chaotic sites and the solitons. (ii) The chaotic sites behave like active sites in usual bond-directed DP, except that when they "die" they can emit a left- or right-moving soliton with probability $c$. (iii) The solitons travel ballistically and die with a probability $d$. (iv) Individual solitons are seen by the chaotic sites as inactive. (v) Upon collision two solitons generate a chaotic site; this is the only way by which solitons enhance the activity. A typical spacetime plot of our model is shown in Fig. 4a, showing the same qualitative behavior as the CML in the soliton-rich regime: finite size clusters of activity surrounded by clouds of solitons.

Mean-field equations. - The rate equations for our stochastic model are

$$
\begin{aligned}
& \dot{S}=c C-S^{2}-d S, \\
& \dot{C}=r C+S^{2}-u C^{2},
\end{aligned}
$$

where $C$ and $S$ are the densities of chaotic sites and of solitons. Pure DP corresponds to the equation $\dot{C}=r C-$ $u C^{2}$. These mean-field equations display both a first- and second-order transition, and the nature of the transition is governed by $z \equiv(d / c)^{2} u$; the change to first-order behav-

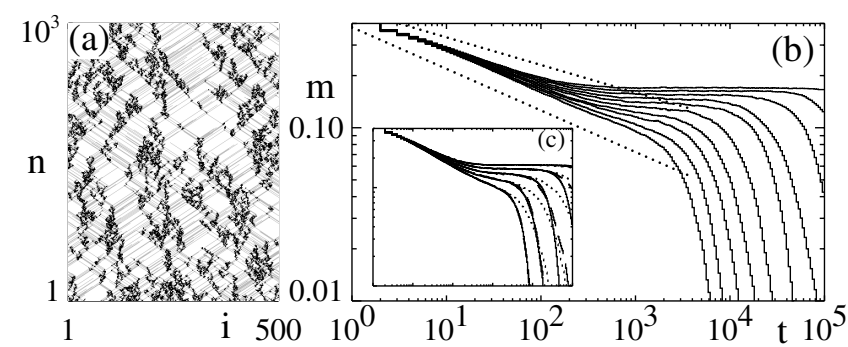

FIG. 4. (a) Soliton dominated dynamics in the stochastic model for $d=0.01, c=0.3$, and $p=0.55$. (b) Average activity $m$ for $c=0.1, d=0.01$, and $p=0.612, \ldots, 0.621$ for an ensemble of 20 systems of size $2 \times 10^{4}$. The dotted curves are for comparison and have slopes 0.159 and 0.24 . (c) Comparison of activity for system sizes 200 (2000 realizations, dotted line), 2000 (200 realizations, dashed line), and 20000 (20 realizations, full curves) for $p=0.613,0.615$, $0.617,0.619$, and 0.621 . For smaller system sizes the final falloff occurring for large $p$ seems more gentle, but clearly the early time behavior and the value of the plateau activity are insensitive to finite size effects.

ior occurs at $z=1$. If $z>1$ (short soliton lifetime) the solitons just renormalize $u$ to $u\left(1-\frac{1}{z}\right)$. When $z<1$ the behavior for positive $r$ (the active phase) is governed by the stable node at $S_{2}^{\star} \approx a\left(z^{-1 / 2}-1\right)$. When $r$ becomes negative this fixed point remains stable and at a finite distance away from the origin; simultaneously, the origin becomes attractive while a saddle at $S=S_{1}^{\star} \approx \frac{d r}{z-1}$ appears close to it. Initial conditions close to the origin will flow there, but initial conditions above the saddle stable manifold will flow to the node. This will go on until $\left(S_{1}^{\star}, C_{1}^{\star}\right)$ and $\left(S_{2}^{\star}, C_{2}^{\star}\right)$ merge in a saddle-node bifurcation at $r=$ $r_{c}(z)<0$ below which the origin becomes globally attractive-clearly a first-order scenario.

Activity in first-order regime.-In Fig. $4 \mathrm{~b}$ we show the evolution of the activity $m$ for our stochastic model in the soliton-rich regime. There are two important features that can be extracted from these data: (i) For a transient period that goes up to time $10^{3}$, it is possible to find values of $p$ such that the decay of $m$ appears to be a power law with a non-DP exponent. For the example shown, a reasonable scaling can be obtained over two decades. However, for this to be real asymptotic scaling, one should be able to have this scaling extend to arbitrary large times; however, the activity curves for sizes 200,2000 , and 20000 precisely curve down at the same time (Fig. 4c); hence there is no hope that increasing the system size extends the time interval over which apparent scaling can be found. (ii) For long times the activity either decays rapidly or first hits a plateau. Clearly, for increasing time the curve of $m$ as function of $p$ will make a sharper and sharper jump, similar to what we found for the CML. In the plateau regime, the qualitative dynamics is as shown in Fig. 4a. We have also checked that for $p=0.621$ the same plateau value is reached for initial activities in the range from 1 to 0.1 ; for initial activities of 0.05 and smaller, there is an initial increase of the activity but the plateau is never reached [14]. 
It remains to be understood whether this soliton-assisted turbulent state can persist forever and thus whether the observed first-order transition is a true thermodynamic transition. One can argue that below the DP threshold [i.e., for negative $r$ in (6)] the active state can be destroyed by creating a sufficiently large laminar hole, so large that the solitons emitted from the edges cannot penetrate through it and collide to create turbulence [25]. Thus the motion of the edges of such a large "droplet" would be driven only by the diffusion of the chaotic sites, which for negative $r$ will cause the laminar region to expand. Something like this is observed for states close to but below the observed transition, where the activity remains almost constant for a long transient time after which it rapidly decays. We have not been able to determine if this transient time actually diverges at the apparent first-order transition point (which would make it a bona fide phase transition). Such an investigation is difficult since for finite lattices the true asymptotic is always the absorbing state.

Discussion.-The overall picture that emerges from our models is that the transition to spatiotemporal intermittency is strongly influenced by coherently traveling solitons, which, even though they have a finite lifetime, change the nature of the transition. Recent work [26] showing that the transition in the damped Kuramoto-Sivashinsky equation appears first order provides additional support for this scenario. Further support comes indirectly from the finding [27] that the Chaté-Manneville model yields critical behavior consistent with DP when it is driven asynchronously, which destroys the solitons. We believe that our findings also explain the nonuniversality observed in these systems, since our stochastic model shows transient behavior which can be fitted convincingly to power laws that vary strongly with the parameters [14]. In general our study highlights that it is far from trivial to decide which are the important degrees of freedom when mapping deterministic to stochastic behavior: apparently innoculous structures may have an unexpected strong effect on the coarse grained dynamics and render the "natural," most simple stochastic models inapplicable.

It is a pleasure to thank Deepak Dhar, Martin Evans, Geoff Grinstein, Martin Howard, and Kent Bækgaard Lauritsen for valuable discussions. M.v.H. acknowledges financial support from the EU under Contract No. ERBFMBICT 972554 and support from CATS at the Niels Bohr Institute.

[1] T. Bohr, M.H. Jensen, G. Paladin, and A. Vulpiani, Dy- namical Systems Approach to Turbulence (Cambridge University Press, Cambridge, 1998).

[2] A. Novick-Cohen and G. I. Sivashinsky, Physica (Amsterdam) 20D, 237 (1986).

[3] H. Chaté and P. Manneville, Phys. Rev. Lett. 58, 112 (1987).

[4] H. Chaté, in Spontaneous Formation of Space-Time Structures and Criticality, edited by T. Riste and D. Sherrington (Kluwer, Dordrecht, 1991), p. 273.

[5] S. Ciliberto and P. Bigazzi, Phys. Rev. Lett. 60, 286 (1988); F. Daviaud, M. Dubois, and P. Bergé, Europhys. Lett. 9, 441 (1989); M. Rabaud, S. Michalland, and Y. Couder, Phys. Rev. Lett. 64, 184 (1990); S. Michalland and M. Rabaud, Physica (Amsterdam) 61D, 197 (1992).

[6] Y. Pomeau, Physica (Amsterdam) 23D, 3 (1986).

[7] G. Grinstein and M.A. Munoz, in Fourth Granada Lectures in Computational Physics, edited by P. Garrido and J. Marro, Lecture Notes in Physics Vol. 493 (Springer, Berlin, 1997), p. 223.

[8] H. Hinrichsen, cond-mat/0001070.

[9] P. Grassberger, Z. Phys. B 47, 365 (1982).

[10] H. K. Janssen, Z. Phys. B 42, 151 (1981).

[11] H. Chaté and P. Manneville, Physica (Amsterdam) 32D, 409 (1988).

[12] P. Grassberger and T. Schreiber, Physica (Amsterdam) 50D, 177 (1991).

[13] E. Domany and W. Kinzel, Phys. Rev. Lett. 53, 311 (1984).

[14] T. Bohr, R. Mikkelsen, M. van Hecke, and M. Ipsen (to be published).

[15] See, e.g., E. Ott, Chaos in Dynamical Systems (Cambridge University Press, Cambridge, 1993).

[16] M. Hénon, Commun. Math. Phys. 50, 69 (1976).

[17] J. M. Houlrik, I. Webman, and M. H. Jensen, Phys. Rev. A 41, 4210 (1990); J. M. Houlrik and M. H. Jensen, Phys. Lett. A 163, 275 (1992); J. M. Houlrik and M. H. Jensen, in Theory and Applications of Coupled Map Lattices, edited by K. Kaneko (Wiley, New York, 1993), p. 95.

[18] I. Jensen, J. Phys. A 29, 7013 (1996).

[19] P. Grassberger and A. de la Torre, Ann. Phys. (N.Y.) 122, 373 (1979).

[20] I. Jensen and R. Dickman, Phys. Rev. E 48, 1710 (1993).

[21] J.F. F. Mendes, R. Dickman, M. Henckel, and M.C. Marques, J. Phys. A 27, 3019 (1994).

[22] M. A. Munoz, G. Grinstein, and R. Dickman, J. Stat. Phys. 91, 541 (1998).

[23] M. A. Munoz, G. Grinstein, and Y. Tu, Phys. Rev. E 56, 5101 (1997).

[24] P. Grassberger, H. Chaté, and G. Rousseau, Phys. Rev. E 55, 2488 (1997).

[25] Rather general considerations in this direction are given in H. Hinrichsen, cond-mat/0006212.

[26] K. R. Elder, J. D. Gunton, and N. Goldenfeld, Phys. Rev. E 56, 1631 (1997).

[27] J. Rolf, T. Bohr, and M. H. Jensen, Phys. Rev. E 57, R2503 (1998). 